\title{
Heterogeneous phenotypic expression of C1QBP variants attributable to variable heteroplasmy of secondary mtDNA deletions and mtDNA copy number
}

josef finsterer ${ }^{1}$

${ }^{1}$ klinikum landstrasse

July 16, 2020

\section{Hosted file}

marchet_let1a.doc available at https://authorea.com/users/343363/articles/470039heterogeneous-phenotypic-expression-of-c1qbp-variants-attributable-to-variableheteroplasmy-of-secondary-mtdna-deletions-and-mtdna-copy-number 